\title{
Current approaches for executing big data science projects - a systematic literature review
}

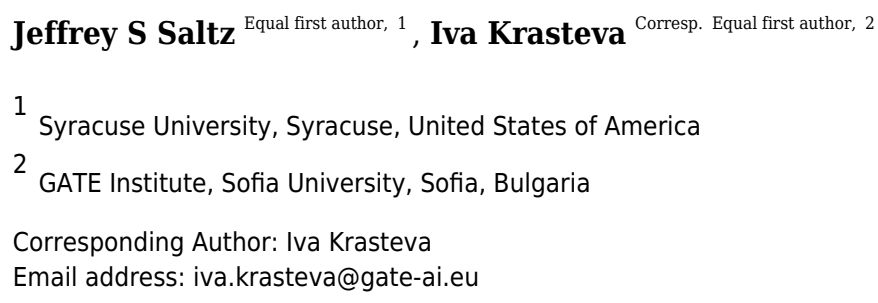

There is an increasing number of big data science projects aiming to create value for organizations by improving decision making, streamlining costs or enhancing business processes. However, many of these projects fail to deliver the expected value. It has been observed that a key reason many data science projects don't succeed is not technical in nature, but rather, the process aspect of the project. The lack of established and mature methodologies for executing data science projects has been frequently noted as a reason for these project failures. To help move the field forward, this study presents a systematic review of research focused on the adoption of big data science process frameworks. The goal of the review was to identify (1) the key themes, with respect to current research on how teams execute data science projects, (2) the most common approaches regarding how data science projects are organized, managed and coordinated, (3) the activities involved in a data science projects life cycle, and (4) the implications for future research in this field. In short, the review identified 68 primary studies thematically classified in six categories. Two of the themes (workflow and agility) accounted for approximately $80 \%$ of the identified studies. The findings regarding workflow approaches consist mainly of adaptations to CRISP-DM (vs entirely new proposed methodologies). With respect to agile approaches, most of the studies only explored the conceptual benefits of using an agile approach in a data science project (vs actually evaluating an agile framework being used in a data science context). Hence, one finding from this research is that future research should explore how to best achieve the theorized benefits of agility. Another finding is the need to explore how to efficiently combine workflow and agile frameworks within a data science context to achieve a more comprehensive approach for project execution. 


\section{Current approaches for executing big data science}

3 projects - a systematic literature review

4

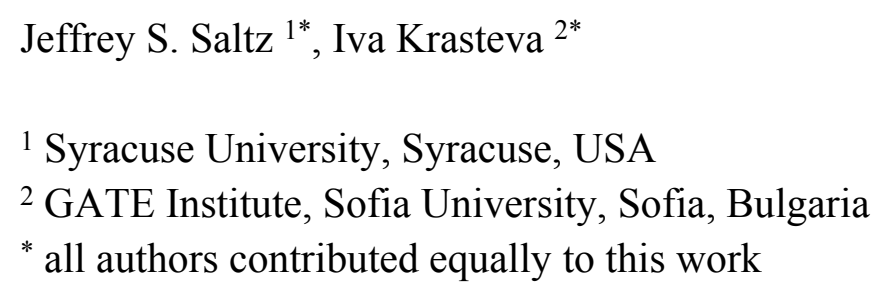

\section{Abstract}

There is an increasing number of big data science projects aiming to create value for organizations by improving decision making, streamlining costs or enhancing business processes. However, many of these projects fail to deliver the expected value. It has been observed that a key reason many data science projects don't succeed is not technical in nature, but rather, the process aspect of the project. The lack of established and mature methodologies for executing data science projects has been frequently noted as a reason for these project failures. To help move the field forward, this study presents a systematic review of research focused on the adoption of big data science process frameworks. The goal of the review was to identify (1) the key themes, with respect to current research on how teams execute data science projects, (2) the most common approaches regarding how data science projects are organized, managed and coordinated, (3) the activities involved in a data science projects life cycle, and (4) the implications for future research in this field. In short, the review identified 68 primary studies thematically classified in six categories. Two of the themes (workflow and agility) accounted for approximately $80 \%$ of the identified studies. The findings regarding workflow approaches consist mainly of adaptations to CRISP-DM (vs entirely new proposed methodologies). With respect to agile approaches, most of the studies only explored the conceptual benefits of using an agile approach in a data science project (vs actually evaluating an agile framework being used in a data science context). Hence, one finding from this research is that future research should explore how to best achieve the theorized benefits of agility. Another finding is the need to explore how to efficiently combine workflow and agile frameworks within a data science context to achieve a more comprehensive approach for project execution.

\section{Introduction}


40 There is an increasing use of big data science across a range of organizations. This means that

41 there is a growing number of big data science projects conducted by organizations. These

42 projects aim to create value by improving decision making, streamlining costs or enhancing

43 business processes.

44 However, many of these projects fail to deliver the expected value (Martinez et al. 2021). For

45 example, VentureBeat (VentureBeats 2019) noted that $87 \%$ of data science projects never make

46 it into production and a NewVantage survey (NewVantage Partners 2019) reported that for 77\%

47 of businesses, the adoption of big data and artificial intelligence (AI) initiatives is a big

48 challenge. A systematic review over the grey and scientific literature has found 21 cases of failed

49 big data projects reported over the last decade (Reggio and Astesiano 2020). This is due, at least

50 in part, to that fact that data science teams generally suffer from immature processes, often

51 relying on trial-and-error and Ad Hoc processes (Bhardwaj et al. 2015)(Gao, Koronios, and Selle

52 2015)(Jeffrey S. Saltz and Shamshurin 2015). In short, big data science projects often do not

53 leverage well-defined process methodologies (Martinez et al. 2021)(Jeffrey S Saltz and Hotz

54 2020). To further emphasize this point, in a survey to data scientists from both industry as well

55 as from not-for-profit organizations, $82 \%$ of the respondents did not follow an explicit process

56 methodology for developing data science projects, and equally important, $85 \%$ of the

57 respondents stated that using an improved and more consistent process would produce more

58 effective data science projects (J Saltz et al. 2018).

59 While a literature review in 2016 did not identify any research focused on improving data

60 science team processes (Jeffrey S. Saltz and Shamshurin 2016), more recently, there has been

61 increase in the studies specifically focused on how to organize and manage big data science

62 projects in more efficient manner (e.g. (Martinez et al. 2021)(Jeffrey S Saltz and Hotz 2020)).

63 With this in mind, this paper presents a systematic review of research focused on the adoption of

64 big data science process frameworks. The purpose is to present an overview of research works,

65 findings, as well as implications for research and practice. This is necessary to identify (1) the

66 key themes, with respect to current research on how teams execute data science projects, (2) the

67 most common approaches regarding how data science projects are organized, managed and

68 coordinated, (3) the activities involved in a data science projects life cycle, and (4) the

69 implications for future research in this field.

70 The rest of the paper is organized as follows: "Background and Related Work" section provides

71 information on big data process frameworks and the key challenges with respect to teams

72 executing big data science projects. In the "Survey Methodology" section, the adopted research

73 methodology is discussed, while the "Results" section presents the findings of the study. The

74 insights from this SLR as well as implications for future research and limitations of the study are

75 highlighted in the "Discussion" section. "Conclusions" section concludes the paper.

76

\section{Background and Related Work}

78 It has been frequently noted that project management (PM) is a key challenge for successfully

79 executing data science projects. In other words, a key reason many data science projects fail is 
80

81

82

83

84

85

86

87

88

89

90

91

92

93

94

95

96

97

98

99

100

101

102

103

104

105

106

107

108

109

110

111

112

113

114

115

116

117

118

119

not technical in nature, but rather, the process aspect of the project (C Ponsard et al. 2017). Furthermore, Espinosa and Armour (Espinosa and Armour 2016) argue that task coordination is a major challenge for data projects. Likewise, Chen, Kazman and Kaziyev (H. M. Chen, Kazman, and Haziyev 2016) conclude that coordination among business analysts, data scientists, system designers, development and operations is a major obstacle that compromises big data science initiatives. Angée et al. (Angée et al. 2018) summarized the challenge by noting that it is important to use an appropriate process methodology, but which, if any, process is the most appropriate is not easy to know.

\section{The importance of using a well-defined process framework}

This data science process challenge, in terms of knowing what process framework to use for data science projects, is important because it has been observed that big data science projects are nontrivial and require well-defined processes (Angée et al. 2018). Furthermore, using a process model or methodology results in higher quality outcomes and avoids numerous problems that decrease the risk of failure in data analytics projects (Mariscal, Marbán, and Fernández 2010). Example problems that occur when a team does not use a process model include the team being slow to share information, deliver the wrong result, and in general, work inefficiently (Gao, Koronios, and Selle 2015)(H.-M. Chen et al. 2017).

\section{The Most Common Framework: CRISP-DM}

The CRoss-Industry Standard Process for Data Mining (CRISP-DM) (Pete et al. 2000) along with Knowledge Discovery in Databases (KDD) (Fayyad, Piatetky-Shapiro, and Smyth 1996), which both were created in the 1990s, are considered 'canonical' methodologies for most of the data mining and data science processes and methodologies (Martinez-Plumed et al. 2019) (Mariscal, Marbán, and Fernández 2010). The evolution of those methodologies can be traced forward to more recent methodologies such as Refined Data Mining Process (Mariscal, Marbán, and Fernández 2010), IBM's Foundational Methodology for Data Science (John B. Rollins 2015) and Microsoft's Team Data Science Process (Microsoft 2020).

However, recent surveys show that when data science teams do use a process, CRISP-DM has been consistently the most commonly used framework and de facto standard for analytics, data mining and data science projects (Martinez-Plumed et al. 2019)(Jeffrey S Saltz and Hotz 2020). In fact, according to many opinion polls, CRISP-DM is the only process framework that is typically known by data science teams (Jeff Saltz, n.d.), with roughly half the respondents reporting to use some version of CRISP-DM.

Specifically, CRISP-DM defines the following six phases:

- Business understanding-includes identification of business objectives and data mining goals

- Data understanding- involves data collection, exploration and validation

- Data preparation- involves data cleaning, transformation and integration

- Modelling - includes selecting modelling technique and creating and assessing models

- $\quad$ Evaluation- evaluates the results against business objectives

- Deployment- includes planning for deployment, monitoring and maintenance. 
120 CRISP-DM allows some high-level iteration between the steps (Gao, Koronios, and Selle 2015).

121 Typically, when a project uses CRISP-DM, the project moves from one phase (such as data

122 understanding) to the next phase (e.g., data preparation). However, as the team deems

123 appropriate, the team can go back to a previous phase. In a sense, one can think of CRISP-DM as

124 a waterfall model for data mining (Gao, Koronios, and Selle 2015).

125 While CRISP-DM is popular, and CRISP-DM's phased based approach is helpful to describe

126 what the team should do, there are some limitations with the framework. For example, the

127 framework provides little guidance on how to know when to loop back to a previous phase,

128 iterate on the current phase, or move to the next phase. In addition, CRISP-DM does not

129 contemplate the need for operational support after deployment.

130 The stated need for more research

131 Given that many data science teams do not use a well-defined process and that others use

132 CRISP-DM with known challenges, it is not surprising that there has been a consistent calling for

133 more research with respect to data science team process. For example, in Cao's discussion of

134 Data Science challenges and future directions (Longbing Cao 2017), it was noted that one of the

135 key challenges in analyzing data includes developing methodologies for data science teams.

136 Gupte (Gupte 2018) similarly noted that the best approach to execute data science projects must

137 be studied. However, even with this noted challenge on data science process, there is a well-

138 accepted view that not enough has been written about the solutions to tackle these problems

139 (Martinez et al. 2021).

140 Is there still a need for more research?

141 This lack of research on data science process frameworks was certainly true six years ago, when

142 the need for concise, thorough and validated information regarding the ways data science

143 projects are organized, managed and coordinated was noted (Jeffrey S. Saltz 2015). This need

144 was further clarified when, in a literature review of big data science process research, no papers

145 were found that focused on improving a data science team's process or overall project

146 management (Ransbotham, David, and Prentice 2015). This was also consistent with the view

147 that most big data science research has focused on the technical capabilities required for data

148 science and has overlooked the topic of managing data science projects (J S Saltz and

149 Shamshurin 2016).

150 However, much has happened during the past six years, with respect to research on data science

151 process frameworks. With this in mind, to help move the field forward, this research aims to

152 focus on the following research questions:

153 - RQ1: Has research in this domain increased recently?

154 - RQ2: What are the most common approaches regarding how data science projects are

$155 \quad$ organized, managed and coordinated?

156 RQ3: What are the phases or activities in a data science project life cycle?

157

158

Survey methodology 
159

160

161

162

163

164

165

166

167

168

169

170

171

172

173

174

175

176

177

178

179

180

181

182

183

184

185

186

187

188

189

190

191

192

193

194

195

196

197

While there are many approaches to a literature review, one approach, which is followed in this research, is to combine quantitative and qualitative analysis to provide deeper insights (Joseph et al. 2007). Furthermore, the systematic literature review conducted in this study leveraged the guidelines for performing SLRs suggested by Kitchenham and Charters (Kitchenham and Charters 2007) and the data were collected in a similar manner as described in (Saltz and Dewar 2019). Hence, the SLR process consisted of three phases: planning, conducting and reporting the review. The subsections below present the outcomes of the first two phases, while the results of the review are reported in the next section.

\section{PLANNING THE REVIEW}

In general, systematic reviews address the need to summarize and present the existing information about some phenomenon in a thorough and unbiased manner (Kitchenham and Charters 2007). As previously noted, the need for concise, thorough and validated information regarding the ways data science projects are organized, managed and coordinated is justified by the lack of established and mature methodologies for executing data science projects. This has led to our previously defined research questions, which are the drivers for how we structured our research.

The study search space comprises the following five online sources: ACM Digital Library, IEEEXplore, Scopus, ScienceDirect and Google Scholar. In addition to online sources, the search space might be enriched with reference lists from relevant primary studies and review articles (Kitchenham and Charters 2007). Specifically, the papers that cite the study providing justification for the present research (Jeffrey S. Saltz 2015) and the previous SLR on the subject (Jeffrey S. Saltz and Shamshurin 2016) are added to the study search space.

Our search strategy includes both metadata and full-text searches over the selected online sources. The search phases that were identified after a couple of iterations, cover the two key concepts relevant to the study:

- $\quad$ Data science related terms: ("data science" OR "big data" OR "machine learning”).

- $\quad$ Project execution related terms: ("process methodology" OR "team process" OR "team coordination" OR "project management").

To determine whether a paper should be included in our analysis, the following selection criteria are defined:

- Inclusion criteria:

- Papers that fully or partly include a description of the organization, management or coordination of big data science projects.

- $\quad$ Papers that suggest specific approaches for executing big data science projects.

- $\quad$ Papers that were published after 2015.

- Exclusion criteria:

- $\quad$ Papers that are not written in English

Peer] Comput. Sci. reviewing PDF | (CS-2021:09:65526:1:1:NEW 18 Dec 2021) 
198

199

200

201

202

203

204

205

206

207

208

209

210

211

212

213

214

215

216

217

218

219

220

221

222

223

224

225

226

227

228

229

230

231

232

233

234

235

- $\quad$ Papers that did not focus on data science team process, but rather, focused on using data analytics to improve overall project management processes were excluded.

- $\quad$ Papers that had no form of peer review (e.g. blogs).

- $\quad$ Papers with irrelevant document type such as posters, conference summaries, etc.

Our exclusion of papers that discussed the use of analytics for overall project management considerations was driven by our desire to focus this research on understanding the specific attributes of data science projects, and how different frameworks were, or were not, applicable in the context of a data science project. This does not imply that data science has no role in helping to improve overall project management approaches. In fact, data science can and should add to the field of general project management, but we view this analysis as beyond the scope of our research.

The selection procedure describes how the selection criteria will be applied while conducting the study (Kitchenham and Charters 2007) (Saltz and Dewar 2019). In our case, we planned two selection steps:

- $\quad$ Step1: Title and abstract screen - Initially, after the relevant papers from the search space are identified according to the study search strategy, the selection criteria will be applied considering only the title and the abstracts of the papers. This step is to be executed by the two authors over different sets of identified papers.

- Step2: Full text screen - The full text of the candidate papers will then be reviewed by the two authors independently to identify the final set of primary studies to be included for further data analysis.

The approach for data extraction and synthesis followed in our study is based on the content analysis suggested in (Elo and Kyngäs 2008) (Hsieh and Shannon 2005). After exploring the key concepts used within each of the primary studies, general research themes are to be identified and further analysis of the data with respect to the study research questions is to be performed in both qualitative and quantitative manner.

\section{CONDUCTING THE REVIEW}

The SLR procedure was performed at the beginning of May, 2021. Because of the differences in running the searches over the online sources included in our search space, the identification of research and the first step of the selection procedure for Google Scholar were executed independently from the other digital libraries.

Three searches for the identification of relevant studies were executed over Google Scholar database with the following search strings:

- $\quad$ Search 1, the "data science" search: "data science" AND ("process methodology" OR "team process" OR "team coordination" OR "project management"). 
236

237

238

239

240

241

242

243

244

245

246

247

248

249

250

251

252

253

254

255

256

257

258

259

260

261

262

263

264

265

266

267

268

269

270

271

272

273

274

275

- $\quad$ Search 2, the "machine learning" search: "machine learning" AND ("process methodology" OR "team process" OR "team coordination" OR "project management").

- $\quad$ Search 3, the "big data" search: "big data" AND ("process methodology" OR "team process" OR "team coordination" OR "project management").

Since the number of papers returned after executing the searches were very large, via a snowball sampling approach, only the first 220 papers in each result sets were included for further analysis. The first step of the selection procedure was executed for the unique papers in each of the sets and 48 papers were selected as candidates for primary studies. Table 1 shows the exact number of papers returned after running the searches and the first step of the selection procedure for Google Scholar.

Executing the initial search strings over the digital libraries resulted a vast number of papers (e.g., over 1,500 papers for IEEE Xplore full text). Motivated by the results of the executed searches in Google Scholar, an optimization of the search terms was introduced. Since the ratio of candidate to retrieved papers for the "machine learning" Google Scholar search string was very low and only one paper was selected after the first step of the selection procedure, we removed the term "machine learning" from the initial "Data science related terms" search phrase. The final search string that was used for identification of studies from the digital libraries the was: ("data science" OR "big data" OR "machine learning") AND ("process methodology" OR "team process" OR "team coordination" OR "project management").

Both metadata and full text searches were performed over the four digital libraries:

- $\quad$ ACM Digital Library - full text search.

- IEEEXplore -metadata-based and full text searches.

- $\quad$ Scopus - metadata-based search.

- $\quad$ ScienceDirect - metadata-based search.

When executing the searches, appropriate filters helping to meet inclusion and exclusion criteria for each of the sources were applied where available. We used Mendeley as a reference management tool to help us organize the retrieved papers and to automate the removal of duplicates. A total of 1944 was returned by the searches, from which 1697 were unique papers. After executing the title and abstract screen, 98 papers were selected for candidates for primary studies. The exact numbers of retrieved and candidate papers are presented in Table 2. The numbers shown in the table include papers duplicated across the digital libraries.

The relevant studies search space comprised the papers that cite the two studies which provide the proper justification and relevant background for our research, namely (Jeffrey S. Saltz 2015) and (Jeffrey S. Saltz and Shamshurin 2016). A total of 159 papers were found to cite the two papers. After filtering the papers by screening the titles and abstracts, 64 of those papers were selected for candidate primary studies.

A consolidated list of all the candidate papers which were selected in the previous step of the selection procedure was created. The list included 120 unique papers. After performing the next step of the selection procedure (full text review), 68 papers were selected. These papers

Peer) Comput. Sci. reviewing PDF | (CS-2021:09:65526:1:1:NEW 18 Dec 2021) 
276 comprised the list of primary studies that were further analyzed to provide the answers to our 277 research questions. The steps of the SLR procedure that led to the identification of the primary 278 studies for our study are presented in Fig 1.

279 Following the guidelines by Cruzes and Dybå (Cruzes and Dybå 2011), thematic analysis and 280 synthesis was applied during data extraction and synthesis. We used the integrated approach 281 (Cruzes and Dybå 2011), which employs both inductive and deductive code development, for 282 retrieving the research themes related to the execution of data science projects as well as for 283 defining the categories of workflow approaches and the themes for agile adoption presented in 284 the following section.

285

286

287

288

289

290

291

292

293

294

295

296

297

298

299

300

301

302

303

304

305

306

307

308

309

310

311

312

313

314

315

\section{Results}

This section presents the findings of the SLR with regard to the three research questions defined in the planning phase.

\section{RESEARCH ACTIVITY IN THIS DOMAIN (RQ1)}

As shown in Fig2, there has been an increase in the number of articles published over time. Note that the review was in done in May 2021, so the 2021 year was on pace to have more papers than any other year (i.e., over the full year, 2021 was on pace to have 18+ papers). Furthermore, it is likely that 2020 had a reduction due to COVID.

We also explored publishing outlets. Specifically, Fig3 shows the number of papers for each publisher. IEEE was the most frequent publisher, with 31 (46\%) papers, due in part to a yearly IEEE workshop on this domain, that started in 2015. The next highest publisher was ACM, with 9 papers (13\%).

\section{APPROACHES FOR EXECUTING DATA SCIENCE PROJECTS (RQ2)}

Table 3 provides an overview of the six themes identified, with respect to the approaches for defining and using a data science process framework. The table also shows the relevant primary studies. While the six themes that we identified in our SLR are all relevant to project execution, there was a wide range in the number of papers published for the different themes. The ratio of publications across the different themes provides a high-level view of current research efforts regarding the execution of data science projects.

Below we provide a description for each of the themes, with an expanded focus on the two most popular themes (workflows and agility).

Workflows papers explored how data science projects were organized with respect to the phases, steps, activities and tasks of the execution process (e.g., CRISP-DM's project phases). There were 27 papers in this theme, which is about $40 \%$ of the total number of primary studies. Workflow approaches are discussed in our second research question and a detailed overview of the relevant studies will be provided in the following section.

Agility papers described the adoption of agile approaches and considered specific aspects of project execution such as the need for iterations or how teams should coordination and 
316 collaborate. The high number of papers categorized in the Agility theme (26 out of 68 ) might be 317 due to the successful adoption of agile methodologies in various software development projects.

318 The theme will be covered in the next section since agile adoption is also relevant to our second 319 research question. Seven papers explored both the workflows and agility themes.

320 Process adoption papers discussed the key factors as well as the challenges for a data science

321

322

323

324

325

326

327

328

329

330

331

332

333

334

335

336

337

338

339

340

341

342

343

344

345

346

347

348

349

350

351

352

353

354

355

team to adopt a new process. Specifically, the papers that discussed process adoption considered questions such as acceptance factors (J S Saltz 2017)(Jeffrey S. Saltz 2018)(Jeffrey Saltz and Hotz 2021), project success factors (Soukaina et al. 2019), exploring the application of software engineering practices in the data science context (Jeffrey S. Saltz and Shamshurin 2017), and would deep learning impact a data science teams process adoption (Shamshurin and Saltz 2019a).

General PM papers discussed general project management challenges. These papers did not focus on addressing any data science unique characteristics, but rather, general management challenges such as the team's process maturity (Jeffrey S. Saltz and Shamshurin 2015), the need for collaboration (Mao et al. 2019), the organizational needs and challenges when executing projects (Ramesh and Ramakrishna 2018) and training of human resources (Mullarkey et al. 2019).

Tools focused papers described new tools that could improve the data science team's productivity. Five papers explored how different tools, both custom and commercial, could be used to support various aspects of the execution of the data science projects. The tools explored focused on communication and collaboration (Marin 2019)(Wang et al. 2019), Continuous Integration / Continuous Development (A. Chen et al. 2020), the maintainability of a data science project (J S Saltz et al. 2020) and a tool to improve the coordination of the data science team (Crowston et al. 2021).

Reviews were papers that reported on a SLR for a specific topic related to data science project execution or papers that report on an industry survey. An SLR aiming to find out benefits and challenges on applying CRISP-DM in research studies is presented in (Schröer, Kruse, and Gómez 2021). How different data mining methodologies are adapted in practice is investigated in (Plotnikova, Dumas, and Milani 2020). That literature review covered 207 peer- reviewed and 'grey' publications and identified four adaptation patters and two recurrent purposes for adaptation. Another SLR focused on experience reports and explored the adoption of agile software development methods in data science projects (Krasteva and Ilieva 2020). An extensive critical review over 19 data science methodologies is presented in (Martinez et al. 2021). The paper also proposed principles of an integral methodology for data science which should include the three foundation stones: project, team and data \& information management. Professionals with different roles across multiple organizations were surveyed in (J Saltz et al. 2018) about the methodology they used in their data science projects and whether an improved project management process would benefit their results. The two papers that formed the core of our search space of related papers, (Jeffrey S. Saltz 2015) and (Jeffrey S. Saltz and Shamshurin 2016), were also included in the Reviews thematic category.

Peer] Comput. Sci. reviewing PDF | (CS-2021:09:65526:1:1:NEW 18 Dec 2021) 
356

357

358

359

360

361

362

363

364

365

366

367

368

369

370

371

372

373

374

375

376

377

378

379

380

381

382

383

384

385

386

387

388

389

390

391

392

393

394

395

\section{WORKFLOW APPROACHES}

The thematic analysis of the workflows for data science projects revealed that the workflows might be broadly categorized in three groups: (1) standard, (2) new, and (3) adapted workflows. Furthermore, three sub-categories of adapted workflows were synthesized based on the aim of the adoption:

- $\quad$ Specialization - adjustments to standard workflows, which are made to better suit particular big data technology or specific domain.

- Extension - addition of new steps, tasks or activities to extend standard workflow phases.

- $\quad$ Enrichment - extension of the scope of a standard workflow to provide more comprehensive coverage of the project execution activities.

An overview of workflow categories and respective primary studies is presented in Table 4 . Multiple studies of the same workflow are shown in brackets. Most of the workflows use a standard framework as a reference point for specification of both new and adapted workflows. As seen in Table 4, CRISP-DM provides the basis for the majority of the workflow papers. Below we explore each of these categories in more depth.

\section{New Workflows}

While the workflow proposed in (Nancy W. Grady 2016) make use of CRISP-DM activities, a new workflow with four phases, five stages and more than 15 activities was designed to accommodate big data technologies and data science activities. Providing a more focused technology perspective, (Amershi et al. 2019) proposes a nine-stage workflow for integrating machine learning into application and platform development. Uniting the advantages of experimentation and iterative working along with a greater understanding of the user requirements, a novel approach for data projects is proposed in (Ahmed, Dannhauser, and Philip 2019). The suggested workflow consists of 3 stages and 7 steps and integrates the principles of the Lean Start-up method and design thinking with CRISP-DM activities. The workflows in (Dutta and Bose 2015) and (Shah, Gochtovtt, and Baldini 2019) are designed and used in companies, and integrate strategic perspective with planning, management and implementation.

\section{Standard Workflows}

Three of the primary studies reported on using CRISP-DM in student projects and compared and contracted the adoption of different methodologies (e.g. CRISP-DM, Scrum and Kanban) for executing data science projects.

\section{Workflow Specializations}

Specialization category is the smallest of the three adaption sub-categories. Two of the workflows in this category were based on CRISP-DM and were specialized for sequence analysis (Kalgotra and Sharda 2016) or anomaly detection (Schwenzfeier and Gruhn 2018). In addition, a revised KDD procedure model for time-series data was proposed in (Vernickel et al. 2019).

\section{Workflow Extensions}


396

397

398

399

400

401

402

403

404

405

406

407

408

409

410

411

412

413

414

415

416

417

418

419

420

421

422

423

424

425

426

427

428

429

430

431

432

433

434

435

An extension to CRISP-DM for knowledge discovery on social networks was specified as a seven-stage workflow that can be applied in different domains intersecting with social network platforms (Asamoah and Sharda 2019). While this workflow extended CRISP-DM for big data, the workflows in (Christophe Ponsard, Touzani, and Majchrowski 2017) and (Qadadeh and Abdallah 2020) added additional workflow steps focused on identification of data value and business objectives. An extension to KDD for public healthcare was proposed in (Silva, Saraee, and Saraee 2019). The suggested workflow implies user-friendly techniques and tools to help healthcare professionals use data science in their daily work. By performing a SLR of recent developments in KD process models, (Jeroen Baijens and Helms 2019) proposes relevant adjustments of the steps and tasks of the Refined Data Mining Process (Mariscal, Marbán, and Fernández 2010). The IBM's Analytics Solutions Unified Method for Data Mining/predictive analytics (ASUM-DM) is extended in (Angée et al. 2018) for a specific use case in the banking sector with focus on big data analytics, prototyping and evaluation. A software engineering lifecycle process for big data projects is proposed in (lin and Huang 2017) as an extension to the ISO/IEC standard 15288:2008.

\section{Workflow Enrichments}

There were several papers that extend CRISP-DM in different dimensions. The studies in (Kolyshkina and Simoff 2019) and (Fahse, Huber, and van Giffen 2021) addressed two important aspects of ML solutions - interpretability and bias, respectively. They suggested new activities and methods integrated in CRISP-DM steps for satisfying desired interpretability level and for bias prevention and mitigation. A novel approach for custom workflow creation from a flexible and comprehensive Data Science Trajectory map of activities was suggested in (Martinez-Plumed et al. 2019). The approach is designed to address the diversity of data science projects and their exploratory nature. The workflow presented in (Kordon 2020) proposes improvements to CRISP-DM in several areas - maintenance and support, knowledge acquisition and project management. Scheduling, roles and tools are integrated with CRISP-DM in a methodology, presented in (Costa and Aparicio 2020). Checkpoints and synchronization are used in the proposed in (Yamada and Peran 2017) Analytics Governance Framework to facilitate communication and coordination between the client and the data science team. Collaboration is the primary focus in (Zhang, Muller, and Wang 2020), in which a basic workflow is extended with collaborative practices, roles and tools.

\section{AGILE APPROACHES}

As shown in Table 5, there were 26 papers that focused on the need for agility within data science projects. Only $31 \%$ of the papers actually reported on teams using an agile approach. The rest of the papers, $69 \%$ (18 of the 26 papers), were conceptual in nature. These conceptual papers explained why it makes sense that a framework should be helpful for a data science project but provided no examples that the framework actually helps a data science team.

Specifically, the vast majority of the papers ( 15 papers), explored the potential benefits of agility for data science projects. These papers were labeled general agility papers since they did not 
436 explicitly support any specific agile approach, but rather, noted the benefits teams should get by 437 adopting an agile framework. The expected benefits of agility typically focused on the need for 438 multiple iterations to support the exploratory nature of data science projects, especially since the 439 outcomes are uncertain. This would allow teams to adjust their future plans based on the results 440 of their current iteration.

441 Two papers discussed the potential benefits of Scrum. However, five papers reported on the 442 difficulty teams encountered when they actually tried to use Scrum. Often times, issues arose due 443 to the challenge in accurately estimating how long a task would take to complete. This issue of 444 task estimation impacted the team's ability to determine what work items could fit into a sprint.

445 Two other papers reported on the use of Scrum within data science team, but both of those 446 papers did not describe in depth how the team used Scrum, nor if there were any benefits or 447 issues due to their use of Scrum.

448 Finally, one paper discussed the conceptual benefits of using a lean approach and a different 449 paper reported on the challenge in using Kanban (which can be thought as supporting both 450 agility and lean principles). That paper explored the need for the process master role, similar to 451 the Scrum Master role in Scrum.

452

\section{COMBINED APPROACHES}

454 The seven papers that covered both the workflow and agility themes presented a more comprehensive methodology for project execution. Several proposed new frameworks (N W Grady, Payne, and Parker 2017)(Christophe Ponsard, Touzani, and Majchrowski 2017)(C Ponsard et al. 2017)(Ahmed, Dannhauser, and Philip 2019). All of the newly proposed frameworks defined a new workflow (typically based on CRISP-DM), and also suggested that

460

461

462

463

464

465

466

467

468

469

470

471

472

473

474 the project do iterations and focus on creating a minimal viable product (MVP). However, there was no consensus on if the iterations should be time-boxed or capability based. Furthermore, there no consensus on how to integrate the data science life cycle into each iteration. In fact, two papers didn't explicitly address this question (Christophe Ponsard, Touzani, and Majchrowski 2017)(C Ponsard et al. 2017) and another article implied that something should be done for each phase in each sprint (N W Grady, Payne, and Parker 2017). Yet another article suggested that maybe some iterations focus on a specific phase and other iterations might focus on more than one phase (Ahmed, Dannhauser, and Philip 2019).

Three articles analyzed existing frameworks, including both workflow and agile frameworks (Jeffrey Saltz, Shamshurin, and Crowston 2017)(J Saltz, Heckman, and Shamshurin 2017)(Shah, Gochtovtt, and Baldini 2019). For both of these articles, there was not explicit discussion on how to integrate workflow frameworks with agile frameworks.

\section{DATA SCIENCE PROJECT LIFE CYCLE ACTIVITIES (RQ3)}

Table 6 shows a synthesized overview of the life cycle phases mentioned in the workflow papers, presented above. This table also shows the number (and percentage) of papers that mention a 
475 specific data science life cycle phase. One can note that the most common phases are the CRISP-

476 DM phases.

477

478 Discussion

479 The section presents further analysis on the findings of the study, highlighting the insights and 480 implications for future research as well as exploring several validity threats.

481 Insights and implications for future research

482 The analysis of the information extracted for each primary study provided interesting insights on

483 how data science projects are currently organized, managed and executed. The findings

484 regarding categories of workflows confirm the trend observed in (Plotnikova, Dumas, and Milani

485 2020) of the large number of adaptations of workflow frameworks (vs proposing new

486 methodologies). While CRISP-DM is reported to be the most widely used framework for data

487 science projects (e.g.(Jeffrey S Saltz and Hotz 2020)), the adaptions of CRISP-DM in data

488 science projects are much more commonly reported in the research literature, which raises the

489 question if teams are adapting CRISP-DM, when they are using it within their project.

490 Most of the agility papers were conceptual in nature, and many of the other papers reported on

491 issues when using Scrum. Hence, more research is needed to explore how to achieve the

492 theorized benefits of agility, perhaps by adapting Scrum or using a different framework.

493 Combining workflow approaches with agile frameworks within a data science context is a way to 494 achieve an integral framework for project execution. However, more research is needed on how 495 to combine these two approaches. For example, the research presented in (Martinez et al. 2021)

496 over the 19 methodologies for data science projects determined that only four of them could be 497 classified as integral according to the criteria defined in the study. Specifying new data science 498 methodologies that cover different aspects of project execution (e.g. team coordination, data and 499 system engineering, stakeholder collaboration) is a promising direction for future research.

500 To explore if the life cycle activities mentioned in the workflow papers have changed over time, 501 we conducted a comparative analysis with a similar SLR in which 23 data mining process 502 models are compared based on process steps (Rotondo and Quilligan 2020). As all of the papers 503 from the previous SLR were prior to 2018, comparing the two SLR's provides a way to see if the 504 usage of different phases has changed over time. It was observed that the use of an exploratory 505 phase (Data Analysis / Exploration) was increasing, while the model interpretation and 506 explanation phase (Interpret / Explain) was decreasing. The last is perhaps due to these tasks 507 being integrated into the evaluation phase.

508 Validity threats

509 Several limitations of the study present potential threats to its validity. One limitation is that the 510 SLR was based on a specific set of search strings. It is possible a different search string could 511 have identified other interesting articles. Adding an additional search space based on citations of 512 relevant studies tried to mitigate the impact of this potential threat.

513 Another limitation is that while authors explored ACM Digital Library, IEEEXplore, Scopus, 514 ScienceDirect and Google Scholar databases, which index high impact journals and conference 
515 papers from IEEE, ACM, SpringerLink, and Elsevier, it is possible that some relevant articles 516 from other publication outlets could have been missed. In addition, the grey literature was not

517 analyzed. This literature could have provided additional insights on the adoption of data science

518 approaches in industrial settings. Yet another limitation is that the analysis and synthesis were

519 based on qualitative content analysis and thematic synthesis of the selected articles by the

520 research team. The authors tried to minimize the subjectivity of researchers' interpretation by

521 cross-checking papers to reduce bias.

522

523

\section{Conclusions}

524 This study presents a systematic review of research focused on the adoption of big data science

525

526

527

528

529

530

531

532

533

534

535

536

537

538

539

540

541

542

543

544

545

546

547

548

\section{References}

550 Ahmed, Bakhtiyar, Thomas Dannhauser, and Nada Philip. 2019. "A Lean Design Thinking

551 Methodology (LDTM) for Machine Learning and Modern Data Projects." 2018 10th Computer

552 Science and Electronic Engineering Conference, CEEC 2018 - Proceedings, 11-14.

553

process frameworks. The study shows that research on how data science projects are organized, managed and executed has increased significantly during the last six years. Furthermore, the review identified 68 primary studies and thematically classified these studies in six key themes, with respect to current research on how teams execute data science projects (workflows, agility, process adoption, general PM, tools, and reviews). CRISP-DM was the most common workflow discussed, and the different adaption patterns of CRISP-DM - specializations, extensions and enrichments, were the most common approaches for specifying and using adjusted workflows for data science projects.

However, standardized approaches explicitly designed for the data science context were not identified, and hence, is a gap in current research and practice. Similarly, with respect to agile approaches, more research is needed to explore how and if the conceptual benefits of agility noted in many of the identified papers can actually be achieved in practice. In addition, another direction for future research is to explore combining workflow and agile approaches into a more comprehensive framework that covers different aspects of project execution.

The current study can be enhanced and extended in three directions. First, the search space could be expanded by using the snowballing technique (Wohlin 2014) for identification of relevant articles. Some of the primary studies identified in the current study can be used as seed papers in a future execution of the procedure. Second, conducting a multivocal literature review (Garousi, Felderer, and Mäntylä 2016) including grey literature can complement the results of the study by collecting more experience reports and real-world adoptions from industry. Finally, future research could explore if the process used should vary based on different industries, or if, the appropriate data science process is independent of the specific industry project context. https://doi.org/10.1109/CEEC.2018.8674234.

Peer) Comput. Sci. reviewing PDF | (CS-2021:09:65526:1:1:NEW 18 Dec 2021) 
554 Al-Jaroodi, Jameela, Brandon Hollein, and Nader Mohamed. 2017. "Applying Software 555 Engineering Processes for Big Data Analytics Applications Development." In 2017 IEEE 7th 556 Annual Computing and Communication Workshop and Conference (CCWC), 1-7. IEEE. 557 https://doi.org/10.1109/CCWC.2017.7868456.

558 Amershi, Saleema, Andrew Begel, Christian Bird, Robert DeLine, Harald Gall, Ece Kamar, 559 Nachiappan Nagappan, Besmira Nushi, and Thomas Zimmermann. 2019. "Software Engineering 560 for Machine Learning: A Case Study.” In 2019 IEEE/ACM 41st International Conference on 561 Software Engineering: Software Engineering in Practice (ICSE-SEIP), 291-300. IEEE.

562 https://doi.org/10.1109/ICSE-SEIP.2019.00042.

563 Angée, Santiago, Silvia I. Lozano-Argel, Edwin N. Montoya-Munera, J.-D. Juan-David David 564 Ospina-Arango, Marta S. Tabares-Betancur, Santiago Angee, Silvia I. Lozano-Argel, Edwin N. 565 Montoya-Munera, J.-D. Juan-David David Ospina-Arango, and Marta S. Tabares-Betancur.

566

567

568

569

570

571

572

573

574

575

576

577

578

579

580

581

582

583

584

585

586

587

588

589

590

591

592

593 2018. "Towards an Improved ASUM-DM Process Methodology for Data \& Analytics Projects." International Conference on Knowledge Management in Organizations 877 (January 2019): 61324. https://doi.org/10.1007/978-3-319-95204-8.

Asamoah, Daniel Adomako, and Ramesh Sharda. 2019. "CRISP-ESNeP: Towards a Data-Driven Knowledge Discovery Process for Electronic Social Networks." Journal of Decision Systems 28 (4): 286-308. https://doi.org/10.1080/12460125.2019.1696614.

B, Timo Aho, Outi Sievi-korte, Terhi Kilamo, and Sezin Yaman. 2020. Demystifying Data Science Projects: A Look on the People and Process of Data. International Conference on Product-Focused Software Process Improvement (PROFES). Vol. 1. Springer International Publishing. https://doi.org/10.1007/978-3-030-64148-1.

Baijens, J, R Helms, and D Iren. 2020. "Applying Scrum in Data Science Projects.” In Proceedings - 2020 IEEE 22nd Conference on Business Informatics, CBI 2020, 1:30-38. https://doi.org/10.1109/CBI49978.2020.00011.

Baijens, J, R Helms, and R Kusters. 2020. "Data Analytics Project Methodologies: Which One to Choose?" In ACM International Conference Proceeding Series, 41-47. https://doi.org/10.1145/3437075.3437087.

Baijens, Jeroen, and Remko W. Helms. 2019. "Developments in Knowledge Discovery Processes and Methodologies: Anything New?" In 25th Americas Conference on Information Systems, AMCIS 2019.

Becker, David K. 2017. "Predicting Outcomes for Big Data Projects: Big Data Project Dynamics (BDPD): Research in Progress.” In Proceedings - 2017 IEEE International Conference on Big Data, Big Data 2017, 2018-Janua:2320-30. https://doi.org/10.1109/BigData.2017.8258186. Bhardwaj, Anant, Souvik Bhattacherjee, Amit Chavan, Amol Deshpande, Aaron J. Elmore, Samuel Madden, and Aditya Parameswaran. 2015. "DataHub: Collaborative Data Science \& Dataset Version Management at Scale." CIDR 2015 - 7th Biennial Conference on Innovative Data Systems Research.

Chen, Andrew, Andy Chow, Aaron Davidson, Arjun DCunha, Ali Ghodsi, Sue Ann Hong, Andy Konwinski, et al. 2020. "Developments in MLflow." In Proceedings of the Fourth International 
594 Workshop on Data Management for End-to-End Machine Learning, 1-4. DEEM'20. New York, 595 NY, USA: ACM. https://doi.org/10.1145/3399579.3399867.

596 Chen, H.-M, R Kazman, R Schütz, and F Matthes. 2017. "How Lufthansa Capitalized on Big

597 Data for Business Model Renovation.” MISQ Executive 16: 19-34.

598 Chen, Hong Mei, Rick Kazman, and Serge Haziyev. 2016. "Agile Big Data Analytics for Web599 Based Systems: An Architecture-Centric Approach.” Proceedings of the Annual Hawaii

600 International Conference on System Sciences 2016-March (3): 5378-87.

601 https://doi.org/10.1109/HICSS.2016.665.

602 Costa, Carlos J., and Joao Tiago Aparicio. 2020. "POST-DS: A Methodology to Boost Data

603 Science." Iberian Conference on Information Systems and Technologies, CISTI 2020-June

604 (June). https://doi.org/10.23919/CISTI49556.2020.9140932.

605 Crowston, Kevin, Jeffery Saltz, Niraj Sitaula, and Yatish Hegde. 2021. "Evaluating MIDST, A

606 System to Support Stigmergic Team Coordination.” Proc. ACM Hum.-Comput. Interact. 5

607 (CSCW1). https://doi.org/10.1145/3449110.

608 Cruzes, Daniela S., and Tore Dybå. 2011. "Recommended Steps for Thematic Synthesis in

609 Software Engineering." International Symposium on Empirical Software Engineering and

610 Measurement, no. 7491: 275-84. https://doi.org/10.1109/esem.2011.36.

611 Dabrowski, Peter. 2021. "Project Management for a Machine Learning Project." In Machine

612 Learning and Data Science in the Oil and Gas Industry, edited by Patrick Bangert, 129-51. Gulf

613 Professional Publishing. https://doi.org/https://doi.org/10.1016/B978-0-12-820714-7.00007-8.

614 Demigha, Souâd. 2019. “Agile Projects and Big Data.” In Proceedings of the International

615 Conference on Intellectual Capital, Knowledge Management and Organisational Learning,

616 ICICKM, edited by Munir R Dumay J. Guthrie J., 2019-Decem:88-96. Academic Conferences

617 and Publishing International Limited. https://doi.org/10.34190/IKM.19.025.

618 Dharmapal, S R, and K T Sikamani. 2016. "Big Data Analytics Using Agile Model.” In

619 International Conference on Electrical, Electronics, and Optimization Techniques, ICEEOT

620 2016, 1088-91. Institute of Electrical and Electronics Engineers Inc.

621 https://doi.org/10.1109/ICEEOT.2016.7754854.

622 Dutta, Debprotim, and Indranil Bose. 2015. "Managing a Big Data Project: The Case of Ramco

623 Cements Limited." International Journal of Production Economics 165: 293-306.

624 https://doi.org/https://doi.org/10.1016/j.ijpe.2014.12.032.

625 Elo, Satu, and Helvi Kyngäs. 2008. “The Qualitative Content Analysis Process.” Journal of

626 Advanced Nursing 62 (1): 107-15. https://doi.org/10.1111/j.1365-2648.2007.04569.x.

627 Espinosa, J. Alberto, and Frank Armour. 2016. "The Big Data Analytics Gold Rush: A Research

628 Framework for Coordination and Governance." Proceedings of the Annual Hawaii International

629 Conference on System Sciences 2016-March: 1112-21.

630 https://doi.org/10.1109/HICSS.2016.141.

631 Fahse, Tobias, Viktoria Huber, and Benjamin van Giffen. 2021. "Managing Bias in Machine

632 Learning Projects." 16th International Conference on Wirtschaftsinformatik (WI).

633 https://www.alexandria.unisg.ch/262449/. 
634 Fayyad, Usama, Gregory Piatetky-Shapiro, and Padhraic Smyth. 1996. "The KDD Process for 635 Extracting Useful Knowledge from Volumes of Data." Communication of the ACM 39 (11): 27 63634.

637 Franková, P, M Drahošová, and P Balco. 2016. "Agile Project Management Approach and Its

638 Use in Big Data Management.” In Procedia Computer Science, edited by Shakshuki E., 83:576639 83. Elsevier. https://doi.org/10.1016/j.procs.2016.04.272.

640 Gao, Jing, Andy Koronios, and Sven Selle. 2015. "Towards a Process View on Critical Success

641 Factors in Big Data Analytics Projects.” 2015 Americas Conference on Information Systems, 642 AMCIS 2015, 1-14.

643 Garousi, Vahid, Michael Felderer, and Mika V. Mäntylä. 2016. “The Need for Multivocal

644 Literature Reviews in Software Engineering: Complementing Systematic Literature Reviews

645 with Grey Literature.” ACM International Conference Proceeding Series 01-03-June (June).

646 https://doi.org/10.1145/2915970.2916008.

647 Grady, N W, J A Payne, and H Parker. 2017. "Agile Big Data Analytics: AnalyticsOps for Data

648 Science.” In 2017 IEEE International Conference on Big Data (Big Data), 2331-39.

649 https://doi.org/10.1109/BigData.2017.8258187.

650 Grady, Nancy W. 2016. "KDD Meets Big Data." In 2016 IEEE International Conference on Big 651 Data (Big Data), 1603-8. https://doi.org/10.1109/BigData.2016.7840770.

652 Gupte, Aishwarya. 2018. "Determining Critical Success Factors.” Purdue University.

653 https://www.proquest.com/openview/e92a2045a2dee3fef988de6f294a9f08/1?cbl=18750\&pq-

654 origsite $=$ gscholar.

655 Hassani, Rachida, Younès El Bouzekri El Idrissi, and Abdellah Abouabdellah. 2018. "Digital

656 Project Management in the Era of Digital Transformation." In Proceedings of the 2018

657 International Conference on Software Engineering and Information Management - ICSIM2018,

658 98-103. New York, New York, USA: ACM Press. https://doi.org/10.1145/3178461.3178472.

659 Hsieh, Hsiu-Fang, and Sarah E Shannon. 2005. "Three Approaches to Qualitative Content

660 Analysis." Qualitative Health Research 15 (9): 1277-88.

661 https://doi.org/10.1177/1049732305276687.

662 John B. Rollins. 2015. "Foundational Methodology for Data Science." IBM Analytics, 1-4.

663 Joseph, Damien, Kok-Yee Ng, Christine Koh, and Soon Ang. 2007. "Turnover of Information

664 Technology Professionals: A Narrative Review, Meta-Analytic Structural Equation Modeling,

665 and Model Development.” MIS Q. 31 (3): 547-577.

666 Kalgotra, Pankush, and Ramesh Sharda. 2016. "Progression Analysis of Signals: Extending

667 CRISP-DM to Stream Analytics.” Proceedings - 2016 IEEE International Conference on Big

668 Data, Big Data 2016, 2880-85. https://doi.org/10.1109/BigData.2016.7840937.

669 Kitchenham, BA, and S. Charters. 2007. "Guidelines for Performing Systematic Literature

670 Reviews in Software Engineering.” Technical Report 2(3), EBSE-2007-01,.

671 Kolyshkina, Inna, and Simeon Simoff. 2019. "Interpretability of Machine Learning Solutions in

672 Industrial Decision Engineering." In Communications in Computer and Information Science,

673 1127 CCIS:156-70. https://doi.org/10.1007/978-981-15-1699-3_13. 
674 Kordon, Arthur K. 2020. "The AI-Based Data Science Workflow.” In Applying Data Science, 675 189-202. Cham: Springer International Publishing. https://doi.org/10.1007/978-3-030-3637567686.

677 Krasteva, Iva, and Sylvia Ilieva. 2020. "Adopting Agile Software Development Methodologies

678 in Big Data Projects - a Systematic Literature Review of Experience Reports.” In 2020 IEEE

679 International Conference on Big Data (Big Data), 2028-33. IEEE.

680 https://doi.org/10.1109/BigData50022.2020.9378118.

681 Larson, Deanne, and Victor Chang. 2016. "A Review and Future Direction of Agile, Business

682 Intelligence, Analytics and Data Science." International Journal of Information Management 36

683 (5): 700-710. https://doi.org/https://doi.org/10.1016/j.ijinfomgt.2016.04.013.

684 lin, Y.-T., and S.-J. Huang. 2017. "The Design of a Software Engineering Lifecycle Process for

685 Big Data Projects." IT Professional 20 (1): 45-52.

686 https://doi.org/10.1109/MITP.2017.265105546.

687 Longbing Cao, Usama Fayyad. 2017. "Data Science : Challenges and Directions."

688 Communications of the ACM Vol. 60 (No. 8): 59-68.

689 http://delivery.acm.org/10.1145/3020000/3015456/p59-

690 cao.pdf?ip $=108.240 .47 .215 \& \mathrm{id}=3015456 \&$ acc $=$ OPEN\&key=4D4702B0C3E38B35.4D4702B0C

691 3E38B35.6978DEEF473775AF.6D218144511F3437\&CFID $=791759728 \&$ CFTOKEN=8785245

692 4\&_acm_=1501772575_4b9f4ec3dac2f9c27d6decb5123e617d.

693 Mao, Yaoli, Dakuo Wang, Michael Muller, Kush R Varshney, Ioana Baldini, Casey Dugan, and

694 Aleksandra Mojsilović. 2019. "How Data ScientistsWork Together With Domain Experts in

695 Scientific Collaborations: To Find The Right Answer Or To Ask The Right Question?” Proc.

696 ACM Hum.-Comput. Interact. 3 (GROUP). https://doi.org/10.1145/3361118.

697 Maria, Rene Esteves, Luiz Antonio Rodrigues Junior, Luiz Eduardo Guarino De Vasconcelos,

698 Adriano Fonseca Mancilha Pinto, Paulo Takachi Tsoucamoto, Henrique Nunweiler Angelim

699 Silva, Airton Lastori, Adilson Marques Da Cunha, and Luiz Alberto Vieira Dias. 2015.

700 "Applying Scrum in an Interdisciplinary Project Using Big Data, Internet of Things, and Credit

701 Cards." In 2015 12th International Conference on Information Technology - New Generations,

702 67-72. IEEE. https://doi.org/10.1109/ITNG.2015.17.

703 Marin, Ivan. 2019. "Data Science and Development Team Remote Communication: The Use of 704 the Machine Learning Canvas." In 2019 ACM/IEEE 14th International Conference on Global

705 Software Engineering (ICGSE), 18-21. IEEE. https://doi.org/10.1109/ICGSE.2019.00018.

706 Mariscal, Gonzalo, Óscar Marbán, and Covadonga Fernández. 2010. “A Survey of Data Mining

707 and Knowledge Discovery Process Models and Methodologies." Knowledge Engineering

708 Review 25 (2): 137-66. https://doi.org/10.1017/S0269888910000032.

709 Martinez-Plumed, Fernando, Lidia Contreras-Ochando, Cesar Ferri, Jose Hernandez Orallo,

710 Meelis Kull, Nicolas Lachiche, Maria Jose Ramirez Quintana, et al. 2019. "CRISP-DM Twenty

711 Years Later: From Data Mining Processes to Data Science Trajectories." IEEE Transactions on

712 Knowledge and Data Engineering 4347 (c): 1. https://doi.org/10.1109/TKDE.2019.2962680. 
713 Martinez, Iñigo, Elisabeth Viles, I G Olaizola, and Igor G. Olaizola. 2021. "Data Science

714 Methodologies: Current Challenges and Future Approaches.” Big Data Research 24: 100183.

715 https://doi.org/10.1016/j.bdr.2020.100183.

716 Microsoft. 2020. "What Is the Team Data Science Process?” 2020.

717 https://docs.microsoft.com/en-us/azure/architecture/data-science-process/overview.

718 Mullarkey, M T, A R Hevner, T Grandon Gill, and K Dutta. 2019. "Citizen Data Scientist: A

719 Design Science Research Method for the Conduct of Data Science Projects." Lecture Notes in

720 Computer Science (Including Subseries Lecture Notes in Artificial Intelligence and Lecture

721 Notes in Bioinformatics) 11491 LNCS: 191-205. https://doi.org/10.1007/978-3-030-19504-

722 5_13.

723 NewVantage Partners. 2019. "Big Data and AI Executive Survey 2019."

$724 \mathrm{https} / / / \mathrm{www} . t c s . c o m /$ content/dam/tcs-bts/pdf/insights/Big-Data-Executive-Survey-2019-

725 Findings-Updated-010219-1.pdf.

726 Pete, Chapman, Clinton Julian, Kerber Randy, Khabaza Thomas, Reinartz Thomas, Shearer

727 Colin, and Rudiger Wirth. 2000. “Crisp-Dm 1.0.” CRISP-DM Consortium.

728 Plotnikova, V, M Dumas, and F Milani. 2020. "Adaptations of Data Mining Methodologies: A

729 Systematic Literature Review.” PeerJ Computer Science 6: 1-43. https://doi.org/10.7717/PEERJ-

730 CS.267.

731 Ponsard, C, A Majchrowski, S Mouton, and M Touzani. 2017. "Process Guidance for the

732 Successful Deployment of a Big Data Project: Lessons Learned from Industrial Cases.” In

733 IoTBDS 2017 - Proceedings of the 2nd International Conference on Internet of Things, Big Data

734 and Security, 350-55. https://doi.org/10.5220/0006357403500355.

735 Ponsard, Christophe, Mounir Touzani, and Annick Majchrowski. 2017. "Combining Process

736 Guidance and Industrial Feedback for Successfully Deploying Big Data Projects." Open Journal

737 of Big Data (OJBD) 3 (1): 26-41. http://www.ronpub.com/ojbd.

738 Qadadeh, Wafa, and Sherief Abdallah. 2020. "An Improved Agile Framework for Implementing

739 Data Science Initiatives in the Government." Proceedings - 3rd International Conference on

740 Information and Computer Technologies, ICICT 2020, 24-30.

741 https://doi.org/10.1109/ICICT50521.2020.00012.

742 Ramesh, B, and A Ramakrishna. 2018. "Unified Business Intelligence Ecosystem: A Project

743 Management Approach to Address Business Intelligence Challenges.” In 2018 Portland

744 International Conference on Management of Engineering and Technology (PICMET), 1-10.

745 https://doi.org/10.23919/PICMET.2018.8481744.

746 Ransbotham, Sam, Kiron David, and Pamela Kirk Prentice. 2015. "Minding the Analytics Gap."

747 MIT Sloan Management Review, 2015. https://sloanreview.mit.edu/article/minding-the-

748 analytics-gap/.

749 Reggio, G, and E Astesiano. 2020. "Big-Data/Analytics Projects Failure: A Literature Review."

750 In Proceedings - 46th Euromicro Conference on Software Engineering and Advanced

751 Applications, SEAA 2020, 246-55. https://doi.org/10.1109/SEAA51224.2020.00050. 
752 Rotondo, Anna, and Fergus Quilligan. 2020. "Evolution Paths for Knowledge Discovery and

753 Data Mining Process Models.” SN Computer Science 1 (2): 1-19.

754 https://doi.org/10.1007/s42979-020-0117-6.

755 Saltz, J, R Heckman, and I Shamshurin. 2017. "Exploring How Different Project Management

756 Methodologies Impact Data Science Students." In Proceedings of the 25th European Conference

757 on Information Systems, ECIS 2017, 2939-48. Association for Information Systems.

758 https://www.scopus.com/inward/record.uri?eid=2-s2.0-

$75985058023852 \&$ partnerID=40\&md5=95248678a298270f33b1e15a21cfe095.

760 Saltz, J, N Hotz, D Wild, and K Stirling. 2018. "Exploring Project Management Methodologies

761 Used within Data Science Teams." In Americas Conference on Information Systems 2018:

762 Digital Disruption, AMCIS 2018.

763 Saltz, J S. 2017. “Acceptance Factors for Using a Big Data Capability and Maturity Model.” In

764 Proceedings of the 25th European Conference on Information Systems, ECIS 2017, 2602-12.

765 Saltz, J S, R Heckman, K Crowston, and Y Hegde. 2020. "Midst: An Enhanced Development

766 Environment That Improves the Maintainability of a Data Science Analysis." International

767 Journal of Information Systems and Project Management 8 (3): 5-22.

768 https://doi.org/10.12821/ijispm080301.

769 Saltz, J.S., Dewar, N. 2019. "Data science ethical considerations: a systematic literature review

770 and proposed project framework”. Ethics and Information Technology. 21, 197-208.

771 Saltz, J S, and I Shamshurin. 2016. "Big Data Team Process Methodologies: A Literature

772 Review and the Identification of Key Factors for a Project's Success.” In 2016 IEEE

773 International Conference on Big Data (Big Data), 2872-79.

774 https://doi.org/10.1109/BigData.2016.7840936.

775 Saltz, Jeff. n.d. "CRISP-DM Is Still the Most Popular Framework for Executing Data Science

776 Projects.” https://www.datascience-pm.com/crisp-dm-still-most-popular/.

777 Saltz, Jeffrey, and Nicholas Hotz. 2021. "Factors That Influence the Selection of a Data Science

778 Process Management Methodology: An Exploratory Study." Proceedings of the 54th Hawaii

779 International Conference on System Sciences 0: 949-59.

780 https://doi.org/10.24251/hicss.2021.116.

781 Saltz, Jeffrey S. 2015. "The Need for New Processes, Methodologies and Tools to Support Big

782 Data Teams and Improve Big Data Project Effectiveness." Proceedings - 2015 IEEE

783 International Conference on Big Data, IEEE Big Data 2015, 2066-71.

784 https://doi.org/10.1109/BigData.2015.7363988.

785 . 2018. "Identifying the Key Drivers for Teams to Use a Data Science Process

786 Methodology." In 26th European Conference on Information Systems: Beyond Digitization -

787 Facets of Socio-Technical Change, ECIS 2018.

788 Saltz, Jeffrey S., and Ivan Shamshurin. 2015. "Exploring the Process of Doing Data Science via

789 an Ethnographic Study of a Media Advertising Company." Proceedings - 2015 IEEE

790 International Conference on Big Data, IEEE Big Data 2015, 2098-2105.

791 https://doi.org/10.1109/BigData.2015.7363992. 
792

793

794

795

796

797

798

799

800

801

802

803

804

805

806

807

808

809

810

811

812

813

814

815

816

817

818

819

820

821

822

823

824

825

826

827

828

829

830

. 2016. "Big Data Team Process Methodologies: A Literature Review and the Identification of Key Factors for a Project's Success." In 2016 IEEE International Conference on Big Data (Big Data), 2872-79. https://doi.org/10.1109/BigData.2016.7840936.

- 2017. "Does Pair Programming Work in a Data Science Context? An Initial Case Study." Proceedings - 2017 IEEE International Conference on Big Data, Big Data 2017 2018Janua: 2348-54. https://doi.org/10.1109/BigData.2017.8258189.

- 2019. "Achieving Agile Big Data Science: The Evolution of a Team's Agile Process Methodology." Proceedings - 2019 IEEE International Conference on Big Data, Big Data 2019, 3477-85. https://doi.org/10.1109/BigData47090.2019.9005493.

Saltz, Jeffrey S, and Robert R Heckman. 2018. "A Scalable Methodology to Guide Student Teams Executing Computing Projects." ACM Transactions on Computing Education 18 (2): 119. https://doi.org/10.1145/3145477.

Saltz, Jeffrey S, and Nicholas Hotz. 2020. "Identifying the Most Common Frameworks Data Science Teams Use to Structure and Coordinate Their Projects." In 2020 IEEE International Conference on Big Data (Big Data), 2038-42. IEEE.

https://doi.org/10.1109/BigData50022.2020.9377813.

Saltz, Jeffrey, Ivan Shamshurin, and Kevin Crowston. 2017. "Comparing Data Science Project Management Methodologies via a Controlled Experiment." Proceedings of the 50th Hawaii International Conference on System Sciences (2017), 1013-22.

https://doi.org/10.24251/hicss.2017.120.

Saltz, Jeffrey, and Alex Suthrland. 2019. "SKI: An Agile Framework for Data Science." Proceedings - 2019 IEEE International Conference on Big Data, Big Data 2019, 3468-76. https://doi.org/10.1109/BigData47090.2019.9005591.

Schröer, Christoph, Felix Kruse, and Jorge Marx Gómez. 2021. "A Systematic Literature Review on Applying CRISP-DM Process Model." Procedia Computer Science 181 (2019): 526-34. https://doi.org/10.1016/j.procs.2021.01.199.

Schwenzfeier, Nils, and Volker Gruhn. 2018. "Towards a Practical Process Model for Anomaly Detection Systems." In Proceedings of the 1st International Workshop on Software Engineering for Cognitive Services, 41-44. SE4COG '18. New York, NY, USA: ACM. https://doi.org/10.1145/3195555.3195568.

Shah, Samir, Alexander Gochtovtt, and Greg Baldini. 2019. "Importance of Project Management in Business Analytics: Academia and Real World." In , 81-94. https://doi.org/10.1007/978-3319-93299-6_6.

Shamshurin, Ivan, and Jeffrey Saltz. 2019a. "Will Deep Learning Change How Teams Execute Big Data Projects?" In Proceedings - 2018 IEEE International Conference on Big Data, Big Data 2018, 2813-17. IEEE. https://doi.org/10.1109/BigData.2018.8622337.

Shamshurin, Ivan, and Jeffrey S. Saltz. 2019b. "Using a Coach to Improve Team Performance When the Team Uses a Kanban Process Methodology." International Journal of Information Systems and Project Management 7 (2): 61-77. https://doi.org/10.12821/ijispm070204.

Peer) Comput. Sci. reviewing PDF | (CS-2021:09:65526:1:1:NEW 18 Dec 2021) 
831 Silva, C, M Saraee, and M Saraee. 2019. "Data Science in Public Mental Health: A New

832 Analytic Framework." In Proceedings - IEEE Symposium on Computers and Communications.

833 Vol. 2019-June. Institute of Electrical and Electronics Engineers Inc.

834 https://doi.org/10.1109/ISCC47284.2019.8969723.

835 Singla, Kushal, Joy Bose, and Chetan Naik. 2018. "Analysis of Software Engineering for Agile

836 Machine Learning Projects.” INDICON 2018 - 15th IEEE India Council International

837 Conference. https://doi.org/10.1109/INDICON45594.2018.8987154.

838 Soukaina, Mouhib, Houda Anoun, Mohammed Ridouani, and Larbi Hassouni. 2019. “A Study of

839 the Factors and Methodologies to Drive Successfully a Big Data Project.” In 2019 Third

840 International Conference on Intelligent Computing in Data Sciences (ICDS), 1-6. IEEE.

841 https://doi.org/10.1109/ICDS47004.2019.8942257.

842 VentureBeats. 2019. "Why Do 87\% of Data Science Projects Never Make It into Production?"

843 2019. https://venturebeat.com/2019/07/19/why-do-87-of-data-science-projects-never-make-it-

844 into-production/.

845 Vernickel, K, J Weber, X Li, J Berg, and G Reinhart. 2019. "A Revised KDD Procedure for the

846 Modeling of Continuous Production in Powder Processing." In 2019 IEEE International

847 Conference on Industrial Engineering and Engineering Management (IEEM), 340-44. IEEE.

848 https://doi.org/10.1109/IEEM44572.2019.8978828.

849 Wang, April Yi, Anant Mittal, Christopher Brooks, and Steve Oney. 2019. "How Data Scientists

850 Use Computational Notebooks for Real-Time Collaboration." Proceedings of the ACM on

851 Human-Computer Interaction 3 (CSCW): 1-30. https://doi.org/10.1145/3359141.

852 Wohlin, Claes. 2014. "Guidelines for Snowballing in Systematic Literature Studies and a

853 Replication in Software Engineering.” ACM International Conference Proceeding Series.

854 https://doi.org/10.1145/2601248.2601268.

855 Yamada, Atsushi, and Michael Peran. 2017. "Governance Framework for Enterprise Analytics

856 and Data.” In 2017 IEEE International Conference on Big Data (Big Data), 3623-31. IEEE.

857 https://doi.org/10.1109/BigData.2017.8258356.

858 Zhang, Amy X, Michael Muller, and Dakuo Wang. 2020. "How Do Data Science Workers

859 Collaborate? Roles, Workflows, and Tools." Proceedings of the ACM on Human-Computer

860 Interaction 4 (CSCW1): 1-23. https://doi.org/10.1145/3392826.

861 
Figure 1

Steps of the SLR procedure for identification of primary studies 


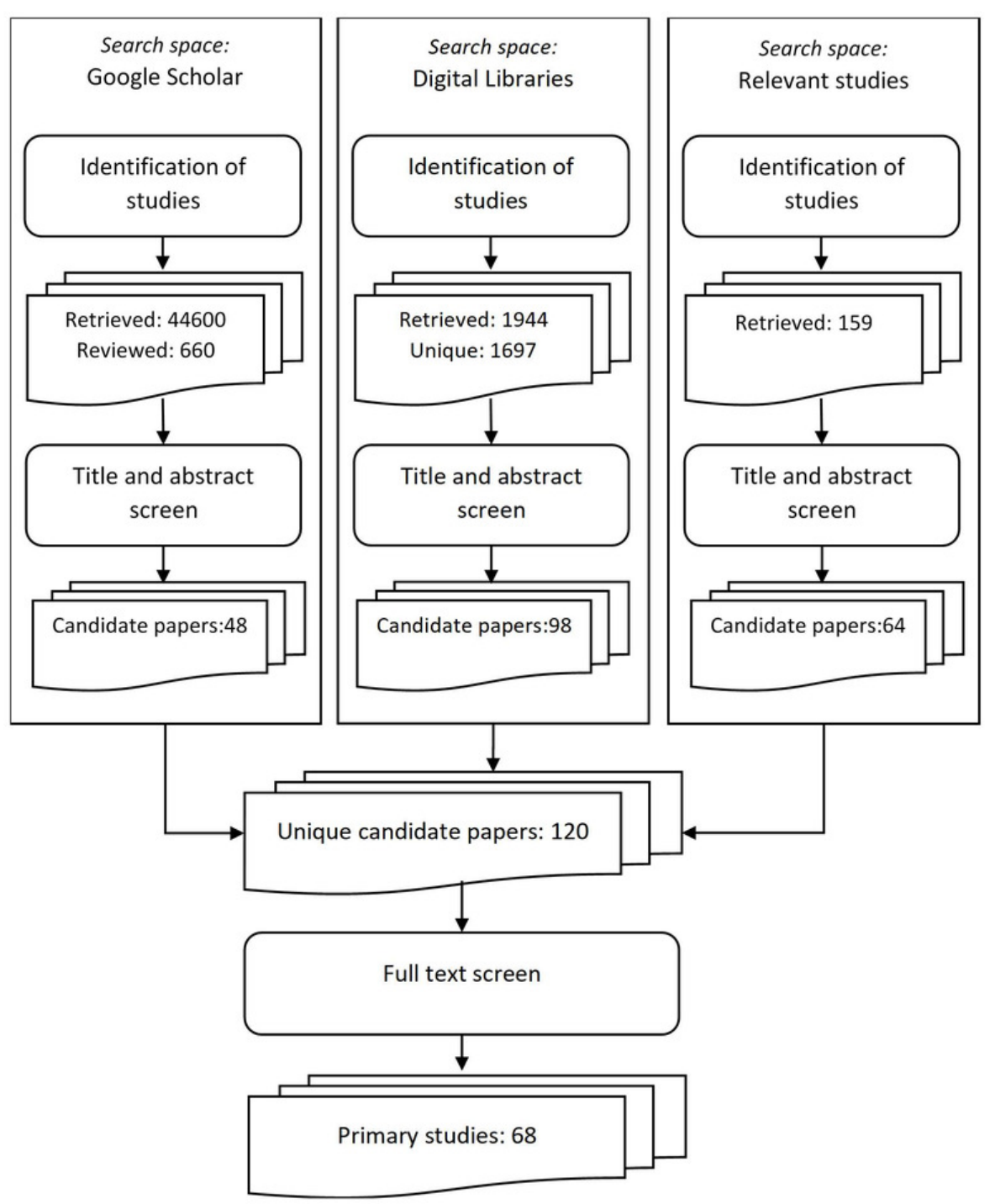


Figure 2

Number of papers per year 


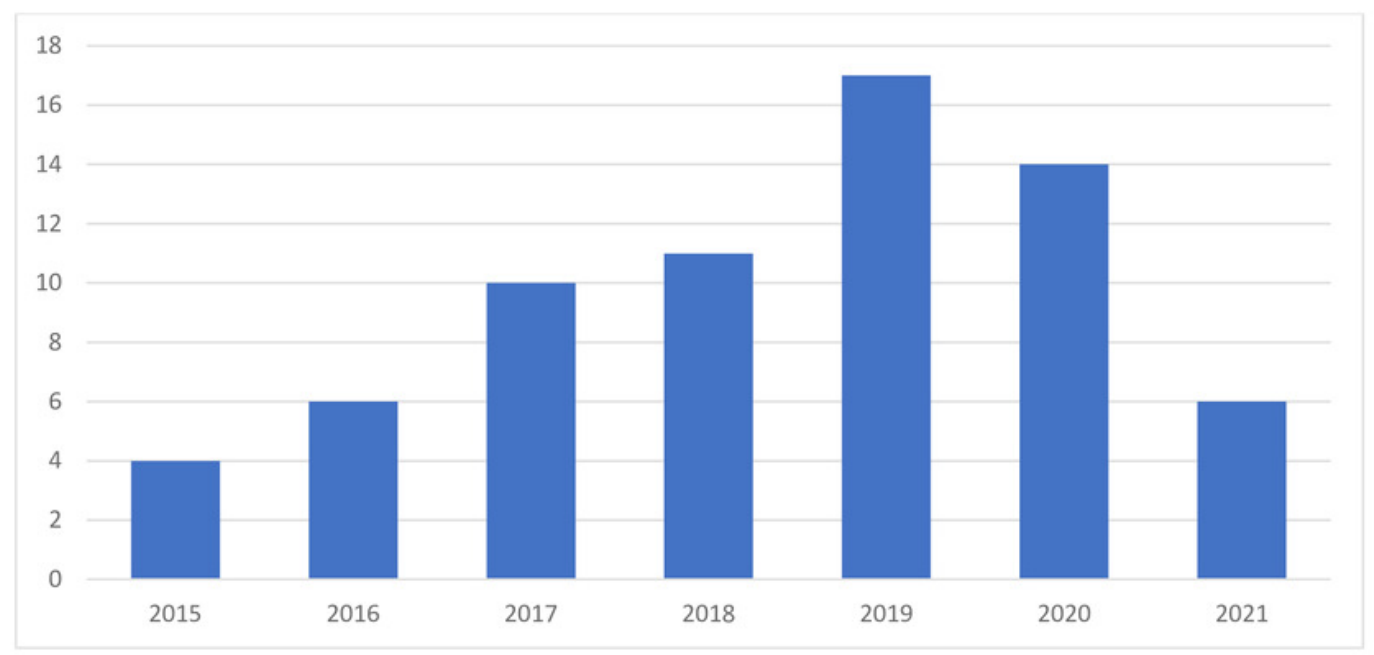


Figure 3

Number of papers for each publisher 


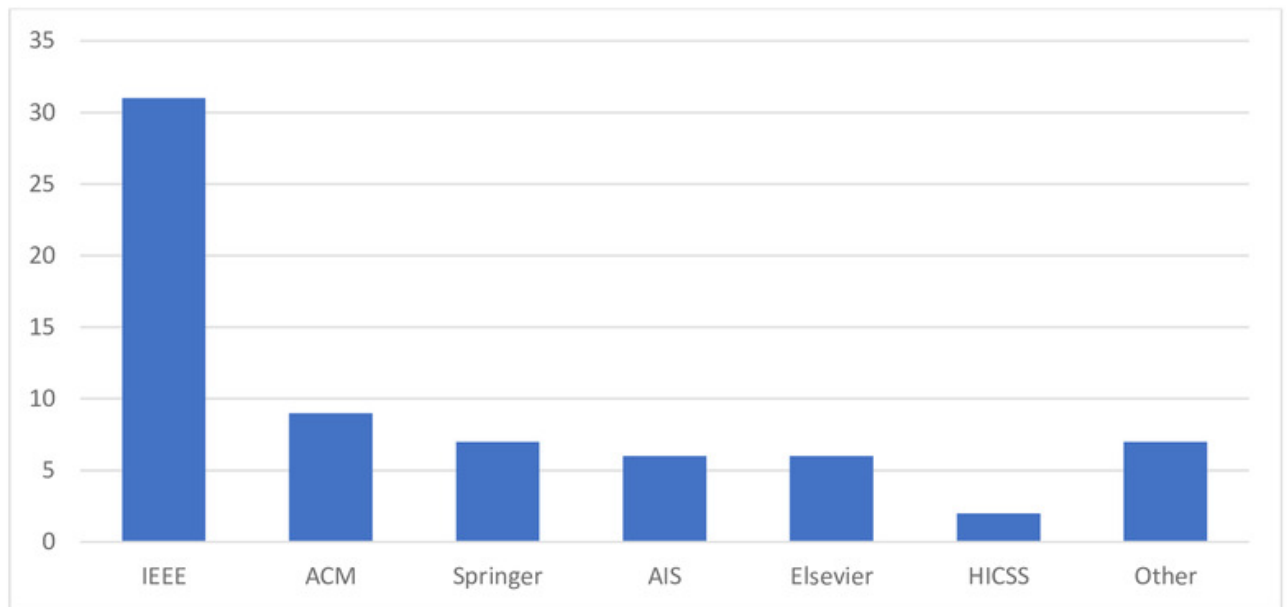




\section{Table $\mathbf{1}$ (on next page)}

Retrieved and candidate papers from Google Scholar 


\begin{tabular}{|l|l|l|}
\hline Search strings & Retrieved papers & Candidate papers \\
\hline "data science" search string & 9,200 (first 220 used) & 37 \\
\hline "machine learning" search string & 17,800 (first 220 used) & 1 \\
\hline "big data" search string & 17,600 (first 220 used) & 10 \\
\hline
\end{tabular}

1 


\section{Table 2 (on next page)}

Retrieved and candidate papers from digital libraries 


\begin{tabular}{|l|l|l|}
\hline Digital library search & Retrieved papers & Candidate papers \\
\hline Scopus: Metadata & 327 & 52 \\
\hline ACM: Full text & 330 & 18 \\
\hline IEEE: All metadata & 197 & 24 \\
\hline IEEE: Full Text & 1,066 & 36 \\
\hline Science Direct: Metadata & 24 & 5 \\
\hline
\end{tabular}

1 


\section{Table 3 (on next page)}

Themes relevant to execution of data science projects 


\begin{tabular}{|l|l|l|}
\hline Theme & Primary studies & Total number \\
\hline Workflows & See Table 4 & 27 \\
\hline Agility & See Table 5 & 26 \\
\hline Process adoption & $\begin{array}{l}\text { (J S Saltz 2017)(Jeffrey S. Saltz 2018)(Jeffrey Saltz } \\
\text { and Hotz 2021) (Soukaina et al. 2019) (Jeffrey S. } \\
\text { Saltz and Shamshurin 2017) (Shamshurin and Saltz } \\
\text { 2019a) }\end{array}$ & 6 \\
\hline General PM & $\begin{array}{l}\text { (Jeffrey S. Saltz and Shamshurin 2015)(Mao et al. } \\
\text { 2019)(Ramesh and Ramakrishna 2018)(Mullarkey } \\
\text { et al. 2019) }\end{array}$ & 4 \\
\hline Tools & $\begin{array}{l}\text { (Marin 2019)(Wang et al. 2019)(A. Chen et al. } \\
\text { 2020)(J S Saltz et al. 2020)(Crowston et al. 2021) }\end{array}$ & 5 \\
\hline Reviews & $\begin{array}{l}\text { (Jeffrey S. Saltz 2015)(Jeffrey S. Saltz and } \\
\text { Shamshurin 2016)(Schröer, Kruse, and Gómez } \\
\text { 2021)(Plotnikova, Dumas, and Milani } \\
\text { 2020)(Krasteva and Ilieva 2020)(Martinez et al. } \\
\text { 2021)(J Saltz et al. 2018) }\end{array}$ & 7 \\
\hline
\end{tabular}




\section{Table 4 (on next page)}

Workflow categories 


\begin{tabular}{|c|c|c|}
\hline Category & $\begin{array}{l}\text { Reference } \\
\text { workflows }\end{array}$ & Primary Studies \\
\hline \multirow[t]{3}{*}{ New } & $\mathrm{N} \backslash \mathrm{A}$ & (Dutta and Bose 2015) (Shah, Gochtovtt, and Baldini 2019) \\
\hline & CRISP-DM & $\begin{array}{l}\text { (Nancy W. Grady 2016)(N W Grady, Payne, and Parker } \\
\text { 2017)(Ahmed, Dannhauser, and Philip 2019) }\end{array}$ \\
\hline & $\begin{array}{l}\text { KDD, } \\
\text { CRISP-DM }\end{array}$ & (Amershi et al. 2019) \\
\hline Standard & CRISP-DM & $\begin{array}{l}\text { (Jeffrey Saltz, Shamshurin, and Crowston 2017)(J Saltz, } \\
\text { Heckman, and Shamshurin 2017)(Jeffrey S Saltz and } \\
\text { Heckman 2018) }\end{array}$ \\
\hline \multirow[t]{2}{*}{ Specialization } & CRISP-DM & (Kalgotra and Sharda 2016)(Schwenzfeier and Gruhn 2018) \\
\hline & KDD & (Vernickel et al. 2019) \\
\hline \multirow[t]{3}{*}{ Extension } & CRISP-DM & $\begin{array}{l}\text { (Christophe Ponsard, Touzani, and Majchrowski 2017)(C } \\
\text { Ponsard et al. 2017)(Asamoah and Sharda 2019)(Qadadeh } \\
\text { and Abdallah 2020) }\end{array}$ \\
\hline & KDD & (Silva, Saraee, and Saraee 2019) \\
\hline & other & $\begin{array}{l}\text { (lin and Huang 2017)(Angée et al. 2018)(Jeroen Baijens and } \\
\text { Helms 2019) }\end{array}$ \\
\hline \multirow[t]{2}{*}{ Enrichment } & CRISP-DM & $\begin{array}{l}\text { (Yamada and Peran 2017)(Martinez-Plumed et al. } \\
\text { 2019)(Kolyshkina and Simoff 2019)(Costa and Aparicio } \\
2020)(\text { Kordon 2020)(Fahse, Huber, and van Giffen 2021) }\end{array}$ \\
\hline & other & (Zhang, Muller, and Wang 2020) \\
\hline
\end{tabular}




\section{Table 5 (on next page)}

Agility themes 


\begin{tabular}{|c|c|c|c|}
\hline Theme & Primary studies & Type & $\begin{array}{l}\text { Total } \\
\text { number }\end{array}$ \\
\hline $\begin{array}{l}\text { Conceptual } \\
\text { Benefits of } \\
\text { Agility }\end{array}$ & $\begin{array}{l}\text { (Franková, Drahošová, and Balco 2016)(Dharmapal } \\
\text { and Sikamani 2016)(N W Grady, Payne, and Parker } \\
\text { 2017)(Al-Jaroodi, Hollein, and Mohamed } \\
\text { 2017)(Christophe Ponsard, Touzani, and } \\
\text { Majchrowski 2017)(Becker 2017)(C Ponsard et al. } \\
\text { 2017)(Hassani, El Bouzekri El Idrissi, and } \\
\text { Abouabdellah 2018) (Demigha 2019)(Shah, } \\
\text { Gochtovtt, and Baldini 2019)(Jeffrey Saltz and } \\
\text { Suthrland 2019)(Reggio and Astesiano 2020)(J } \\
\text { Baijens, Helms, and Kusters 2020)(B et al. } \\
\text { 2020)(Rotondo and Quilligan 2020) }\end{array}$ & Conceptual & $\begin{array}{l}15 \\
(58 \%)\end{array}$ \\
\hline $\begin{array}{l}\text { Challenges } \\
\text { in Scrum }\end{array}$ & $\begin{array}{l}\text { (Jeffrey Saltz, Shamshurin, and Crowston 2017)(J } \\
\text { Saltz, Heckman, and Shamshurin 2017)(Singla, Bose, } \\
\text { and Naik 2018)(Jeffrey S. Saltz and Shamshurin } \\
\text { 2019)(J Baijens, Helms, and Iren 2020) }\end{array}$ & Case Study & $5(19 \%)$ \\
\hline $\begin{array}{l}\text { Scrum is } \\
\text { used }\end{array}$ & (Maria et al. 2015)(Jeffrey S Saltz and Hotz 2020) & Case Study & $2(7 \%)$ \\
\hline $\begin{array}{l}\text { Conceptual } \\
\text { Benefits of } \\
\text { Scrum }\end{array}$ & (Larson and Chang 2016)(Dabrowski 2021) & Conceptual & $2(7 \%)$ \\
\hline $\begin{array}{l}\text { Conceptual } \\
\text { Benefits of } \\
\text { Lean }\end{array}$ & (Ahmed, Dannhauser, and Philip 2019) & Conceptual & $1(4 \%)$ \\
\hline $\begin{array}{l}\text { Challenges } \\
\text { in Kanban }\end{array}$ & (Shamshurin and Saltz 2019b) & Case Study & $1(4 \%)$ \\
\hline
\end{tabular}




\section{Table 6 (on next page)}

Data science life cycle activities 


\begin{tabular}{|l|l|c|}
\hline Theme & Total number & CRISP-DM Phase \\
\hline Readiness assessment & $1(4 \%)$ & \\
\hline Project organization & $5(18 \%)$ & \\
\hline Business understanding & $19(68 \%)$ & \\
\hline Problem identification & $8(29 \%)$ & \\
\hline Data acquisition & $10(36 \%)$ & ß \\
\hline Data understanding & $15(54 \%)$ & \\
\hline Data preparation & $21(75 \%)$ & \\
\hline Feature engineering & $4(14 \%)$ & \\
\hline Data analysis / Exploration & $9(32 \%)$ & \\
\hline Modeling & $25(89 \%)$ & \\
\hline Model refinement & $2(7 \%)$ & \\
\hline Evaluation & $23(82 \%)$ & \\
\hline Interpret / Explain & $2(7 \%)$ & \\
\hline Deployment & $20(71 \%)$ & \\
\hline Business value & $5(18 \%)$ & \\
\hline Monitoring & $2(7 \%)$ & \\
\hline Maintenance & $3(11 \%)$ & \\
\hline
\end{tabular}

